\title{
Extended Reduction Criterion and Lattice States
}

\author{
Fabio Benatti ${ }^{a, b}$, Roberto Floreanini $^{b}$, Alexandra M. Liguori \\ ${ }^{a}$ Dipartimento di Fisica Teorica, Università di Trieste, Strada Costiera 11, \\ 34014 Trieste, Italy \\ ${ }^{b}$ Istituto Nazionale di Fisica Nucleare, Sezione di Trieste, 34100 Trieste, Italy
}

\begin{abstract}
We study a particular class of states of a bipartite system consisting of two 4-level parties. By means of an adapted extended reduction criterion we associate their entanglement properties to the geometric patterns of a planar lattice consisting of 16 points.
\end{abstract}

\section{Introduction}

In recent years, due to the rapid growth of quantum information, communication and computation, the necessity of identifying, quantifying and classifying entangled states as a physical resource has given birth to Quantum Entanglement Theory (see the review [1] and references therein). Though the focus is now shifting to multipartite entanglement, still generic quantum correlations in finite dimensional bipartite systems are not completely understood.

From a mathematical point of view, the lack of exhaustive criteria capable of witnessing the presence of bipartite entanglement, is nothing else but the absence of a complete characterization of positive, but not completely positive maps [2, 3, 4]. Looking for new classes of entangled states [5, 6] and for dedicated entanglement witnesses [7] may thus improve the comprehension of the algebraic structure behind physical phenomena like separability, free and bound entanglement [1].

Recently, an extension of the so-called reduction map [8] has been proposed [9] that is tailor-made for revealing the entanglement of states with a specific structure with respect 
to angular momentum. Subsequently, this map was shown 10 to be a particular instance of a larger class of indecomposable positive maps [2, 3, 4]. In the following, we shall adapt the extended reduction criterion to the study of the class of bipartite states of two 4-level parties which were introduced in [11] and further studied in [12. For reasons that will soon become obvious, these $16 \times 16$ density matrices have been called lattice states.

These states are characterized by subsets of a discrete planar lattice with 16 elements and can be grouped together into equivalence classes characterized by specific geometric patterns. By means of the extended reduction map, we shall associate most of them with specific properties of the corresponding lattice states like that of being separable, NPT-entangled or PPT-entangled. Because of this, besides enriching the phenomenology of entangled states, the class of lattice states may also turn out to be a useful arena for the approach based on the discrete Wigner functions [13, 14, 15, 16]

The plan of the paper is as follows: we shall first introduce the lattice states and their presently known properties; then, we shall improve their classification by using the extended reduction criterion; finally, we shall completely characterize some subclasses of them and discuss which ones need stronger entanglement witnesses than those available so far.

\section{Lattice states}

The construction of lattice states is as follows. Let $\sigma_{\alpha}, 0 \leq \alpha \leq 3$, be the Pauli matrices supplemented with $\sigma_{0}=I_{2}$, the $2 \times 2$ identity matrix, and consider the totally symmetric state

$$
\left|\Psi_{+}^{4}\right\rangle=\frac{1}{2} \sum_{i=1}^{4}|i i\rangle \in \mathbf{C}^{16},
$$

where $\{|i\rangle\}_{i=1}^{4}$ is a fixed orthonormal basis in $\mathbf{C}^{4}$. Denoting the tensor products of pairs of Pauli matrices by

$$
\sigma_{\alpha \beta}:=\sigma_{\alpha} \otimes \sigma_{\beta}
$$

the action of $I_{4} \otimes \sigma_{\alpha \beta}, I_{4}=I_{2} \otimes I_{2}$, on $\left|\Psi_{+}^{4}\right\rangle$ yields an orthonormal basis

$$
\left|\Psi_{\alpha \beta}\right\rangle:=\left(I_{4} \otimes \sigma_{\alpha \beta}\right)\left|\Psi_{+}^{4}\right\rangle \in \mathbf{C}^{16}
$$

with corresponding orthogonal projectors

$$
P_{\alpha \beta}:=\left|\Psi_{\alpha \beta}\right\rangle\left\langle\Psi_{\alpha \beta}\left|=\left(I_{4} \otimes \sigma_{\alpha \beta}\right)\right| \Psi_{+}^{4}\right\rangle\left\langle\Psi_{+}^{4}\right|\left(I_{4} \otimes \sigma_{\alpha \beta}\right) .
$$

Let $L_{16}$ denote the finite square lattice consisting of 16 points labeled by pairs $(\alpha, \beta)$ :

$$
L_{16}:=\{(\alpha, \beta): \alpha, \beta=0,1,2,3\} .
$$

We shall also denote by $\mathcal{C}_{\alpha}$ and $\mathcal{R}_{\beta}$ the columns and rows of the lattice $L_{16}$ :

$$
\mathcal{C}_{\alpha}=\left\{(\alpha, \beta) \in L_{16}: \beta=0,1,2,3\right\}, \quad \mathcal{R}_{\beta}=\left\{(\alpha, \beta) \in L_{16}: \alpha=0,1,2,3\right\}
$$


To every subset $I \subseteq L_{16}$ of cardinality $N_{I}$, we associate a mixed state equidistributed over the orthogonal projectors $P_{\alpha \beta}$ labeled by pairs in $I$ :

$$
\varrho_{I}=\frac{1}{N_{I}} \sum_{(\alpha, \beta) \in I} P_{\alpha \beta} .
$$

We shall refer to the $\varrho_{I}$ as lattice states: they are a particular subclass of the mixed states

$$
\varrho_{\pi}=\sum_{(\alpha, \beta) \in L_{16}} \pi_{\alpha \beta} P_{\alpha \beta}, \quad \pi_{\alpha \beta} \geq 0, \sum_{(\alpha, \beta) \in L_{16}} \pi_{\alpha \beta}=1
$$

that commute with the projectors $P_{\alpha \beta}$.

It turns out that properties of these states like that of being PPT or bound entangled are related to certain specific geometrical patterns of the subsets that label them. Indeed, the following results have been proved (see [12] and [11]):

\section{Proposition 1}

1. A nececessary and sufficient condition for a lattice state $\varrho_{I}$ to be PPT is that for every $(\alpha, \beta) \in L_{16}$ the number of points on $\mathcal{C}_{\alpha}$ and $\mathcal{R}_{\beta}$ different from $(\alpha, \beta)$ and beloging to $I$ be not greater than $N_{I} / 2$. In terms of the characteristic functions $\chi_{I}(\alpha, \beta)=1$ if $(\alpha, \beta) \in I,=0$ otherwise, a lattice state $\varrho_{I}$ is PPT if and only if for all $(\alpha, \beta) \in L_{16}$

$$
\sum_{\delta=0, \delta \neq \beta}^{3} \chi_{I}(\alpha, \delta)+\sum_{\gamma=0, \gamma \neq \alpha}^{3} \chi_{I}(\gamma, \beta) \leq \frac{N_{I}}{2} .
$$

2. A sufficient condition for a PPT lattice state $\varrho_{I}$ to be entangled is that there exists at least a pair $(\alpha, \beta) \in L_{16}$ such that only one point on $\mathcal{C}_{\alpha}$ and $\mathcal{R}_{\beta}$ and different from $(\alpha, \beta)$ belongs to $I$. Equivalently, $\varrho_{I}$ is entangled if a pair $(\alpha, \beta) \in L_{16}$ exists such that

$$
\sum_{\delta=0, \delta \neq \beta}^{3} \chi_{I}(\alpha, \delta)+\sum_{\gamma=0, \gamma \neq \alpha}^{3} \chi_{I}(\gamma, \beta)=1, \quad \text { and } \quad(\alpha, \beta) \notin I .
$$

\section{Remarks 1}

1. A necessary and sufficient condition for PPTness can be worked out for the more general states in (3)); it reads:

$$
\sum_{\delta=0, \delta \neq \beta}^{3} \pi_{\alpha \delta}+\sum_{\gamma=0, \gamma \neq \alpha}^{3} \pi_{\gamma \beta} \leq \frac{1}{2} .
$$


2. From [12], all states $\varrho_{I}$ with $N_{I}=1,2,3,5,7$ are NPT. Therefore in order for $\varrho_{I}$ to be PPT, $N_{I}$ must be equal to $4,6,8$ or 9 or larger.

3. Since the maximum number of points on $\mathcal{C}_{\alpha} \cup \mathcal{R}_{\beta}$ different form $(\alpha, \beta)$ is at most 7 , all states with $N_{I}=14,15,16$ are PPT. Those with $N_{I}=15,16$ are also separable since $N_{I}=16$ means that $\rho_{I}$ is the maximally mixed state, while, for $N_{I}=15, \rho_{I}$ is easily seen to be a separable isotropic state [8, 11].

4. If the condition in (5) is satisfied for one pair $(\alpha, \beta) \in L_{16}$, then $N_{I}=16-6=10$ at the most. In the following, we shall use an entanglement witness which will allow us to add to $I$ the site $(\alpha, \beta)$ from a pair $\left(\mathcal{C}_{\alpha}, \mathcal{R}_{\beta}\right)$ satisfying (15). This will open the possibility of checking the entanglement of lattice states with $N_{I}=11$.

According to the previous proposition, all $\varrho_{I}$ not fulfilling (4) are NPT and thus entangled, while only some of those which are PPT are recognized as entangled by (5)).

As an example of how the geometric picture works, consider the following lattice subsets, where the crosses mark those sites which contribute to $\varrho_{I}$ :

$$
\begin{aligned}
& N_{I}=8 \begin{array}{c|c|c|c|c}
3 & & & & \\
\hline 2 & \times & \times & & \times \\
\hline 1 & \times & \times & & \\
\hline 0 & \times & \times & \times & \\
\hline & 0 & 1 & 2 & 3
\end{array} \\
& N_{I}=10 \begin{array}{cc|c|c|c|c}
3 & \times & \times & \times & \\
\hline 2 & \times & & \times & \\
\hline 1 & & \times & & \times \\
\hline 0 & \times & \times & \times & \\
\hline & 0 & 1 & 2 & 3
\end{array} \\
& N_{I}=8 \begin{array}{c|c|c|c|c}
3 & \times & \times & & \times \\
\hline 2 & \times & & \times & \\
\hline 1 & \times & \times & & \times \\
\hline 0 & & & & \\
\hline & 0 & 1 & 2 & 3
\end{array} \\
& N_{I}=10 \begin{array}{c|c|c|c|c}
3 & \times & \times & & \times \\
\hline 2 & \times & & \times & \\
\hline 1 & & \times & & \times \\
\hline 0 & \times & \times & & \times \\
\hline & 0 & 1 & 2 & 3
\end{array} \\
& N_{I}=10 \begin{array}{c|c|c|c|c}
3 & & \times & \times & \times \\
\hline 2 & \times & \times & \times & \times \\
\hline 1 & & \times & \times & \times \\
\hline 0 & & & & \\
\hline & 0 & 1 & 2 & 3
\end{array} \\
& N_{I}=11 \begin{array}{c|c|c|c|c}
3 & \times & \times & \times & \\
\hline 2 & \times & \times & \times & \times \\
\hline 1 & \times & \times & \times & \\
\hline 0 & & & & \times \\
\hline & 0 & 1 & 2 & 3
\end{array}
\end{aligned}
$$

All subsets above identify PPT lattice states according to (4), but, according to (5), only those in the left column correspond to (bound) entangled states with certainty.

In the following section, we will develop a criterion strictly stronger than (5): it will essentially amount to the removal of the request $(\alpha, \beta) \notin I$ in (5) $)$. This allows us to enrich the class of bound entangled lattice states, proving that also the states on the right hand side are entangled. On the other hand, in Section 3.2, we will further show that some PPT $\varrho_{I}$ which are not recognized as entangled by the stronger criterion are indeed separable. 


\section{Lattice states and the extended reduction criterion}

We adapt the argument of [9] and [10] to the lattice states case by extending the reduction criterion [8] in the following way. We define an antiunitary map $\vartheta_{V}$ by its action on a generic $4 \times 4$ matrix $B \in M_{4}(\mathbf{C}): \vartheta_{V}[B]=V B^{T} V^{\dagger}$, where $T$ denotes transposition with respect to a fixed orthonormal basis and $V: \mathbf{C}^{4} \mapsto \mathbf{C}^{4}$ is unitary and antisymmetric, $V V^{\dagger}=V^{\dagger} V=I_{4}$, $V^{T}=-V$.

It follows that, for all $|\psi\rangle \in \mathbf{C}^{4}$, by expanding with respect to the chosen basis,

$$
\left\langle\psi^{*}|V| \psi\right\rangle=\sum_{j, k=1}^{4} \psi_{j} V_{j k} \psi_{k}=\left\langle\psi^{*}\left|V^{T}\right| \psi\right\rangle=-\left\langle\psi^{*}|V| \psi\right\rangle .
$$

Thus, $\vartheta_{V}[|\psi\rangle\langle\psi|]$ is orthogonal to $|\psi\rangle\langle\psi|$ so that $|\psi\rangle\langle\psi|-\vartheta_{V}[|\psi\rangle\langle\psi|]$ is a projector for all $|\psi\rangle \in \mathbf{C}^{4}$, whence the linear map on $M_{4}(\mathbf{C})$,

$$
\Phi_{V}[B]=(\operatorname{Tr}(B)) I_{4}-B-\vartheta_{V}[B],
$$

preserves positivity.

Remark 2 Since we shall later exhibit PPT lattice states such that $\left(\operatorname{id} \otimes \Phi_{V}\right)\left[\varrho_{I}\right]$ is not positive definite, it turns out that $\Phi_{V}$ is not decomposable, in agreement with [10].

Notice that the request of antisymmetry and the fact that $T\left[\sigma_{2}\right]=-\sigma_{2}$ give the following expansion of $V$ along the tensor products $\sigma_{\alpha \beta}$

$$
V=\sum_{\alpha \neq 2} v_{\alpha 2} \sigma_{\alpha 2}+\sum_{\beta \neq 2} v_{2 \beta} \sigma_{2 \beta}
$$

where $v_{\alpha 2}$ and $v_{2 \alpha}$ are complex coefficients satisfying unitarity constraints.

In order to study the entanglement witnessing power of $\Phi_{V}$ for the lattice states, we shall use that $\vartheta_{V}$ is unitarily equivalent to the transposition and elaborate on the partial action of the latter on the projections $P_{\alpha \beta}$ in (2). In passing, this will provide a proof of (4) alternative to that in [11].

Before proceeding, we make the following useful observations concerning the algebraic relations among the Pauli matrices and the lattice structure.

The products of two Pauli matrices define four $4 \times 4$ hermitian, unitary matrices $\eta^{\alpha}$,

$$
\sigma_{\alpha} \sigma_{\beta}=\sum_{\mu=0}^{3} \eta_{\beta \mu}^{\alpha} \sigma_{\mu}, \quad \eta_{\beta \mu}^{\alpha}=\frac{1}{2} \operatorname{Tr}\left(\sigma_{\alpha} \sigma_{\beta} \sigma_{\mu}\right) .
$$

From the explicit expression of the matrix elements and the cyclicity of the trace operation it follows that

$$
\eta_{\beta \mu}^{\alpha}=\eta_{\alpha \beta}^{\mu}=\eta_{\mu \alpha}^{\beta}, \quad\left(\eta_{\beta \mu}^{\alpha}\right)^{*}=\eta_{\beta \alpha}^{\mu}=\eta_{\mu \beta}^{\alpha} .
$$


The matrices $\eta^{\alpha}$ are thus hermitian and multiplication by $\sigma_{\alpha}$ on the left of both sides of (9) shows that they are also unitary. Explicitly, $\eta^{0}=I_{4}$ while

$$
\eta^{1}=\left(\begin{array}{cccc}
0 & 1 & 0 & 0 \\
1 & 0 & 0 & 0 \\
0 & 0 & 0 & i \\
0 & 0 & -i & 0
\end{array}\right), \eta^{2}=\left(\begin{array}{cccc}
0 & 0 & 1 & 0 \\
0 & 0 & 0 & -i \\
1 & 0 & 0 & 0 \\
0 & i & 0 & 0
\end{array}\right), \eta^{3}=\left(\begin{array}{cccc}
0 & 0 & 0 & 1 \\
0 & 0 & i & 0 \\
0 & -i & 0 & 0 \\
1 & 0 & 0 & 0
\end{array}\right)
$$

Further, given $0 \leq \alpha, \beta \leq 3$ the Pauli matrix satisfying (91) is unique, thus any fixed $\alpha$ determines a map $i_{\alpha}:\{0,1,2,3\} \ni \beta \mapsto i_{\alpha}(\beta) \in\{0,1,2,3\}$ defined by

$$
\sigma_{\alpha} \sigma_{\beta}=\sum_{\mu=0}^{3} \eta_{\beta \mu}^{\alpha} \sigma_{\mu}=\eta_{\beta i_{\alpha}(\beta)}^{\alpha} \sigma_{i_{\alpha}(\beta)}
$$

Multiplying both sides of the above equality by $\sigma_{\alpha}$ and taking their hermitian conjugates, the following useful properties of $i_{\alpha}$ follow from (10):

$$
i_{\alpha}^{2}=\mathrm{id}, \quad i_{\beta}(\alpha)=i_{\alpha}(\beta)
$$

Finally, a useful relation involves the matrix $\eta^{2}$ : one can check that

$$
\left|\eta_{\alpha \beta}^{2}\right|=\delta_{\alpha, \beta \oplus 2} \quad \Longrightarrow \quad i_{2}(\beta)=\beta \oplus 2
$$

where $\oplus$ denotes addition mod 4 .

The action of the partial transposition can now be recast as

$$
(\mathrm{id} \otimes T)\left[P_{\alpha \beta}\right]=\frac{I_{16}}{4}-\frac{1}{2} \sum_{(\mu, \nu) \in L_{16}}\left(\delta_{\alpha, \mu \oplus 2}+\delta_{\beta, \nu \oplus 2}-2 \delta_{\alpha, \mu \oplus 2} \delta_{\beta, \nu \oplus 2}\right) P_{\mu \nu} .
$$

This can be proved using the following facts:

1. by transposing the Pauli matrices one gets

$$
T\left[\sigma_{\alpha}\right]=\sum_{\beta=0}^{3} \varepsilon_{\alpha \beta} \sigma_{\beta} \quad \text { where } \quad \varepsilon=\left[\varepsilon_{\mu \nu}\right]=\left(\begin{array}{cccc}
1 & 0 & 0 & 0 \\
0 & 1 & 0 & 0 \\
0 & 0 & -1 & 0 \\
0 & 0 & 0 & 1
\end{array}\right)
$$

2. partially transposing $P_{00}=\left|\Psi_{+}^{4}\right\rangle\left\langle\Psi_{+}^{4}\right|$ yields the flip $F: A \otimes B \mapsto B \otimes A, A, B \in M_{4}(\mathbf{C})$ :

$$
(\mathrm{id} \otimes T)\left[P_{00}\right]=\frac{1}{4} \sum_{i, j=1}^{4}|i\rangle\langle j|\otimes| j\rangle\langle i|=\frac{F}{4} ;
$$

3. $F$ has the spectral decomposition $F=\sum_{(\alpha, \beta) \in L_{16}} \varepsilon_{\alpha \alpha} \varepsilon_{\beta \beta} P_{\alpha \beta}$. 
Thus one derives

$$
\begin{aligned}
(\mathrm{id} \otimes T)\left[P_{\alpha \beta}\right] & =\frac{1}{4} \sum_{(\mu, \nu) \in L_{16}} \varepsilon_{\mu \mu} \varepsilon_{\nu \nu}\left(I_{4} \otimes \sigma_{\alpha \beta} \sigma_{\mu \nu}\right) P_{00}\left(I_{4} \otimes \sigma_{\mu \nu} \sigma_{\alpha \beta}\right) \\
& =\frac{1}{4} \sum_{(\gamma, \delta),\left(\gamma^{\prime}, \delta^{\prime}\right) \in L_{16}}\left(\eta^{\alpha} \varepsilon \eta^{\alpha}\right)_{\gamma^{\prime} \gamma}\left(\eta^{\beta} \varepsilon \eta^{\beta}\right)_{\delta \delta^{\prime}}\left|\Psi_{\gamma \delta}\right\rangle\left\langle\Psi_{\gamma^{\prime} \delta^{\prime}}\right|
\end{aligned}
$$

whence (15) follows since, using (14), $\left(\eta^{\alpha} \varepsilon \eta^{\alpha}\right)_{\gamma \gamma^{\prime}}=\delta_{\gamma, \gamma^{\prime}}\left(1-2 \delta_{\gamma, \alpha \oplus 2}\right)$.

By means of (15), the action of the partial transposition on lattice states yields

$$
\begin{aligned}
(\mathrm{id} \otimes T)\left[\varrho_{I}\right] & =\frac{I_{16}}{4}-\frac{1}{2 N_{I}} \sum_{(\mu, \nu) \in L_{16}} k_{\mu \nu}^{I} P_{\mu \nu} \quad \text { with } \\
k_{\mu \nu}^{I} & =\sum_{\alpha \neq \mu \oplus 2} \chi_{I}(\alpha, \nu \oplus 2)+\sum_{\beta \neq \nu \oplus 2} \chi_{I}(\mu \oplus 2, \beta),
\end{aligned}
$$

whence the partial action of the extended reduction map (7) gives

$$
\left(\mathrm{id} \otimes \Phi_{V}\right)\left[\varrho_{I}\right]=\frac{1}{2 N_{I}} \sum_{(\mu, \nu) \in L_{16}} k_{\mu \nu}^{I}\left(I_{4} \otimes V\right) P_{\mu \nu}\left(I_{4} \otimes V^{\dagger}\right)-\varrho_{I}
$$

From (16) and (17) the proof of (4) (and of (6) ) easily follows. Moreover, using (18) one gets:

Proposition 2 Let $\kappa$ denote the minimum of $k_{\mu \nu}^{I}$ in (17). Then, a necessary condition for $\Phi_{V}$ to reveal the entanglement of any PPT $\varrho_{I}$ is $\kappa \leq 1$.

Proof: Since unitary transformations do not change an operator's spectrum, it is more convenient to work with the positive, indecomposable map $\tilde{\Phi}_{V}$ defined as follows:

$$
\begin{aligned}
\left(\mathrm{id} \otimes \widetilde{\Phi}_{V}\right)\left[\varrho_{I}\right] & =\left(I_{4} \otimes V^{\dagger}\right)\left[\left(\mathrm{id} \otimes \Phi_{V}\right)\left[\varrho_{I}\right]\right]\left(I_{4} \otimes V\right) \\
& =-\left(I_{4} \otimes V^{\dagger}\right) \varrho_{I}\left(I_{4} \otimes V\right)+\frac{1}{2 N_{I}} \sum_{(\mu, \nu) \in L_{16}} k_{\mu \nu}^{I} P_{\mu \nu}
\end{aligned}
$$

Then, since $\sum_{(\alpha, \beta) \in L_{16}} P_{\alpha \beta}=I_{4}$,

$$
\rho_{I} \leq \frac{I_{16}}{N_{I}} \Longrightarrow\left(\mathrm{id} \otimes \tilde{\Phi}_{V}\right)\left[\varrho_{I}\right] \geq \frac{1}{2 N_{I}} \sum_{(\mu, \nu) \in L_{16}}\left(k_{\mu \nu}^{I}-2\right) P_{\mu \nu}
$$

From the previous result and (17), the extended reduction criterion based on $\Phi_{V}$ may detect entangled PPT lattice states only if 
- $\kappa=0$ : namely, if there exists at least one pair $(\mu, \nu) \in L_{16}$ such that $k_{\mu \nu}^{I}=0$. This is possible if there exist a column $\mathcal{C}_{\mu \oplus 2}$ and a row $\mathcal{R}_{\nu \oplus 2}$ which do not contribute to $I$ except, possibly, for $(\mu \oplus 2, \nu \oplus 2)$;

- $\kappa=1$ : namely, if there exists at least one pair $(\mu, \nu) \in L_{16}$ with $k_{\mu \nu}^{I}=1$. This is possible if there exist a column $\mathcal{C}_{\mu \oplus 2}$ and a row $\mathcal{R}_{\nu \oplus 2}$ that contribute to $I$ with only one element except, possibly, for $(\mu \oplus 2, \nu \oplus 2)$.

\subsection{Entangled PPT Lattice States}

In the following, we will consider the second case and postpone the discussion of the first one to the next section. We start by observing that if $(\mu \oplus 2, \nu \oplus 2)$ does not belong to $I$ then, according to (5), the entanglement of $\rho_{I}$ is revealed by the positive, indecomposable map devised in [12]. We shall show that the extended reduction criterion is able to scoop the bound entangled $\varrho_{I}$ in this case, but also when $(\mu \oplus 2, \nu \oplus 2)$ does belong to $I$, thus being stronger than the map used in [12].

Proposition 3 If $k_{\mu \nu}^{I}=1$ for a column $\mathcal{C}_{\mu \oplus 2}$ and a row $\mathcal{R}_{\nu \oplus 2}$, then

$$
\left\langle\Psi_{\mu \nu}\left|\left(\mathrm{id} \otimes \widetilde{\Phi}_{V}\right)\left[\rho_{I}\right]\right| \Psi_{\mu \nu}\right\rangle<0
$$

independently of whether $(\mu \oplus 2, \nu \oplus 2)$ belongs to $I$ or not.

Proof: Since $\left\langle\Psi_{+}^{4}|A \otimes B| \Psi_{+}^{4}\right\rangle=\operatorname{Tr}\left(A^{T} B\right)$, using the map (12) one gets

$$
\begin{aligned}
\left\langle\Psi_{\mu \nu}\left|\left(I_{4} \otimes V^{\dagger}\right) \varrho_{I}\left(I_{4} \otimes V\right)\right| \Psi_{\mu \nu}\right\rangle & =\frac{1}{N_{I}} \sum_{(\alpha, \beta) \in I}\left|\left\langle\Psi_{+}^{4}\left|I_{4} \otimes\left(\sigma_{\mu \nu} V^{\dagger} \sigma_{\alpha \beta}\right)\right| \Psi_{+}^{4}\right\rangle\right|^{2} \\
& =\frac{1}{4 N_{I}} \sum_{(\alpha, \beta) \in I}\left|\operatorname{Tr}\left(\sigma_{i_{\mu}(\alpha) i_{\nu}(\beta)} V\right)\right|^{2} \\
& =\frac{1}{N_{I}} \sum_{(\alpha, \beta) \in I}\left|v_{i_{\mu}(\alpha) i_{\nu}(\beta)}\right|^{2}
\end{aligned}
$$

where, due to (8) , either $i_{\mu}(\alpha)=0,1,3$ with $i_{\nu}(\beta)=2$ or $i_{\nu}(\beta)=0,1,3$ with $i_{\mu}(\alpha)=2$.

Because of (13) and (14), the first case corresponds to $\beta=\nu \oplus 2$ and $\alpha \neq \mu \oplus 2$ and the second one to $\alpha=\mu \oplus 2$ and $\beta \neq \nu \oplus 2$, whence

$$
\begin{gathered}
\left\langle\Psi_{\mu \nu}\left|\left(I_{4} \otimes V^{\dagger}\right) \varrho_{I}\left(I_{4} \otimes V\right)\right| \Psi_{\mu \nu}\right\rangle=\frac{1}{N_{I}}\left\{\sum_{\alpha \neq \mu \oplus 2} \chi_{I}(\alpha, \nu \oplus 2)\left|v_{i_{\mu}(\alpha) 2}\right|^{2}\right. \\
\left.+\sum_{\beta \neq \nu \oplus 2} \chi_{I}(\mu \oplus 2, \beta)\left|v_{2 i_{\nu}(\beta)}\right|^{2}\right\}
\end{gathered}
$$


whence

$$
\begin{aligned}
\left\langle\Psi_{\mu \nu}\right|(\mathrm{id} \otimes & \left.\widetilde{\Phi}_{V}\right)\left[\varrho_{I}\right]\left|\Psi_{\mu \nu}\right\rangle=\frac{1}{2 N_{I}}\left\{\sum_{\alpha \neq \mu \oplus 2} \chi_{I}(\alpha, \nu \oplus 2)\left(1-2\left|v_{i_{\mu}(\alpha) 2}\right|^{2}\right)\right. \\
& \left.+\sum_{\beta \neq \nu \oplus 2} \chi_{I}(\mu \oplus 2, \beta)\left(1-2\left|v_{2} i_{\nu}(\beta)\right|^{2}\right)\right\} .
\end{aligned}
$$

Suppose that the point of $I$ contributing to $k_{\mu \nu}^{I}=1$ be $(\alpha, \nu \oplus 2)$ on the row $\mathcal{R}_{\nu \oplus 2}$, then $V=\sigma_{i_{\mu}(\alpha) 2}$ yields the result. A similar argument holds if the contributing point is $(\mu \oplus 2, \beta)$ on the column $\mathcal{C}_{\mu \oplus 2}$.

Remark 3 The previous result is not sensitive to whether the point $(\mu \oplus 2, \nu \oplus 2)$ belonging to the column $\mathcal{C}_{\mu \oplus 2}$ and row $\mathcal{R}_{\nu \oplus 2}$ does contribute to $I$ or not. If it does not, the extended reduction criterion reveals all the bound entangled lattice states already revealed by the criterion in [12]; if it does belong to $I$, new bound entangled states not revealed by the latter are seen by the extended reduction map.

\subsection{Separable PPT Lattice States}

By direct inspection, the argument of the previous proposition, by which all bound entangled lattice states with $\kappa=1$ are detectable by the extended reduction criterion, cannot be applied when $\kappa=0$.

It turns out that the case $\kappa=0=k_{\mu \nu}^{I}$ for some $(\mu, \nu) \in L_{16}$ with $(\mu \oplus 2, \nu \oplus 2) \notin I$ can be studied by other means. The following observation turns out to be useful.

Remark 4 As observed in [12], one can operate local unitary transformations transforming lattice states into lattice states without changing either their cardinality $N_{I}$ or their entanglement properties. Therefore, one can actually subdivide the lattice states into equivalence classes by operating on them with local unitaries whose only effect is to map the set of subsets $I \in L_{16}$ of a same cardinality into itself. Indeed, given two $4 \times 4$ matrices $U, V$, the structure of $\left|\Psi_{+}^{4}\right\rangle$ is such that

$$
U \otimes V\left|\Psi_{\alpha \beta}\right\rangle=I_{4} \otimes V \sigma_{\alpha \beta} U^{T}\left|\Psi_{+}^{4}\right\rangle
$$

We distinguish the following cases:

1. Choosing $U=I_{4}$ and $V=\sigma_{\alpha \beta}$, we get $I_{4} \otimes \sigma_{\alpha \beta} P_{\alpha \beta} I_{4} \otimes \sigma_{\alpha \beta}=P_{00}$, so that we can move any chosen column $\mathcal{C}_{\alpha}$ and row $\mathcal{R}_{\beta}$ into $\mathcal{C}_{0}$ and $\mathcal{R}_{0}$.

2. Let $U=U_{1} \otimes U_{2}$ and $V=U_{1}^{*} \otimes U_{2}^{*}$, where $U_{1}$, respectively $U_{2}$ are two unitary $2 \times 2$ matrices that rotate the Pauli matrices $\sigma_{i}, \sigma_{i^{\prime}}$, respectively $\sigma_{j}, \sigma_{j^{\prime}}$, one into the other 
(apart possibly from a - sign) keeping fixed the third one, $\sigma_{i^{\prime \prime}}$, respectively $\sigma_{j^{\prime \prime}}$ (and of course $\left.\sigma_{0}\right)$ :

$$
\begin{aligned}
& U_{1} \sigma_{i} U_{1}^{\dagger}= \pm \sigma_{i^{\prime}}, \quad U_{1} \sigma_{i^{\prime}} U_{1}^{\dagger}=\mp \sigma_{i}, \quad i, i^{\prime} \neq 0 \\
& U_{2} \sigma_{j} U_{2}^{\dagger}= \pm \sigma_{j^{\prime}}, \quad U_{2} \sigma_{j^{\prime}} U_{2}^{\dagger}=\mp \sigma_{j}, \quad j, j^{\prime} \neq 0 .
\end{aligned} \quad \text { and }
$$

Then, any two columns $\mathcal{C}_{i}, \mathcal{C}_{i^{\prime}}$, respectively rows $\mathcal{R}_{j}, \mathcal{R}_{j^{\prime}}$ can be changed one into the other, leaving fixed the columns $\mathcal{C}_{0}, \mathcal{C}_{i^{\prime \prime}}$, respectively the rows $\mathcal{R}_{0}, \mathcal{R}_{j^{\prime \prime}}$ :

$$
\begin{aligned}
V \otimes U P_{i j} V^{\dagger} \otimes U^{\dagger} & =I_{4} \otimes\left(U_{1} \sigma_{i} U_{1}^{\dagger} \otimes U_{2} \sigma_{j} U_{2}^{\dagger}\right) P_{00} I_{4} \otimes\left(U_{1} \sigma_{i} U_{1}^{\dagger} \otimes U_{2} \sigma_{j} U_{2}^{\dagger}\right) \\
& =I_{4} \otimes \sigma_{i^{\prime} j^{\prime}} P_{00} I_{4} \otimes \sigma_{i^{\prime} j^{\prime}}=P_{i^{\prime} j^{\prime}} .
\end{aligned}
$$

3. Finally, choosing $U=I_{4}$ and $V$ the flip operator $\left(V\left|\Psi_{+}^{4}\right\rangle=\left|\Psi_{+}^{4}\right\rangle, V \sigma_{\alpha \beta} V=\sigma_{\beta \alpha}\right)$, one gets:

$$
I_{4} \otimes V P_{\alpha \beta} I_{4} \otimes V=I_{4} \otimes\left(V \sigma_{\alpha \beta} V\right)\left|\Psi_{+}^{4}\right\rangle\left\langle\Psi_{+}^{4}\right| I_{4} \otimes\left(V \sigma_{\alpha \beta} V\right)=P_{\beta \alpha} .
$$

One can thus exchange columns and rows of a subset $I \subseteq L_{16}$ obtaining a new lattice state in the same equivalence class, hence with the same entanglement properties.

The previous considerations allow us to prove the following result.

Proposition 4 If $k_{\mu \nu}^{I}=0$ and $(\mu \oplus 2, \nu \oplus 2)$ does not belong to $I$, all the corresponding PPT states $\varrho_{I}$ are separable.

Proof: The hypothesis means that the column $\mathcal{C}_{\mu \oplus 2}$ and the row $\mathcal{R}_{\nu \oplus 2}$ do not contribute to $I$. Thus 7 points on the square lattice $L_{16}$ must be excluded and at the most 9 elements of $L_{16}$ label $\varrho_{I}$. From Remark 1.2 we know that all states $\varrho_{I}$ with $N_{I}=1,2,3,5,7$ are NPT. Therefore in order for $\varrho_{I}$ to be PPT, $N_{I}$ must be equal to 4, 6, 8 or 9. From Remark 1.4, we also know that all PPT $\varrho_{I}$ with $N_{I}=4$ are separable, in particular those associated with the following graphs (and all those in their equivalence classes as in Remarks 5)
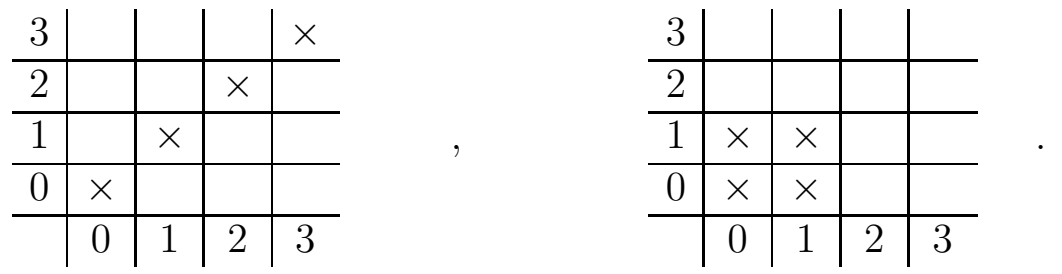

In the following, we shall use these separable rank-4 lattice states to write other lattice states of higher rank as their convex combinations which will thus also result separable; for sake of simplicity, we shall identify graphs with lattice states and the convex decompositions with weighted sums of graphs.

Because of Remark 4, we can always perform a unitary transformation so that the column and row which are assumed to be completely empty correspond to $\mathcal{C}_{0}$ and $\mathcal{R}_{0}$. 
- Case $N_{I}=9$

We have only one possible equivalence class represented by the state $\varrho_{I}^{9}$ associated with the graph

$$
\varrho_{I}^{9}: \quad \begin{array}{c|c|c|c|c}
3 & & \times & \times & \times \\
\hline 2 & & \times & \times & \times \\
\hline 1 & & \times & \times & \times \\
\hline 0 & & & & \\
\hline & 0 & 1 & 2 & 3
\end{array} .
$$

This lattice state can be convexly decomposed in terms of 9 rank-4 PPT (hence separable) lattice states as follows:
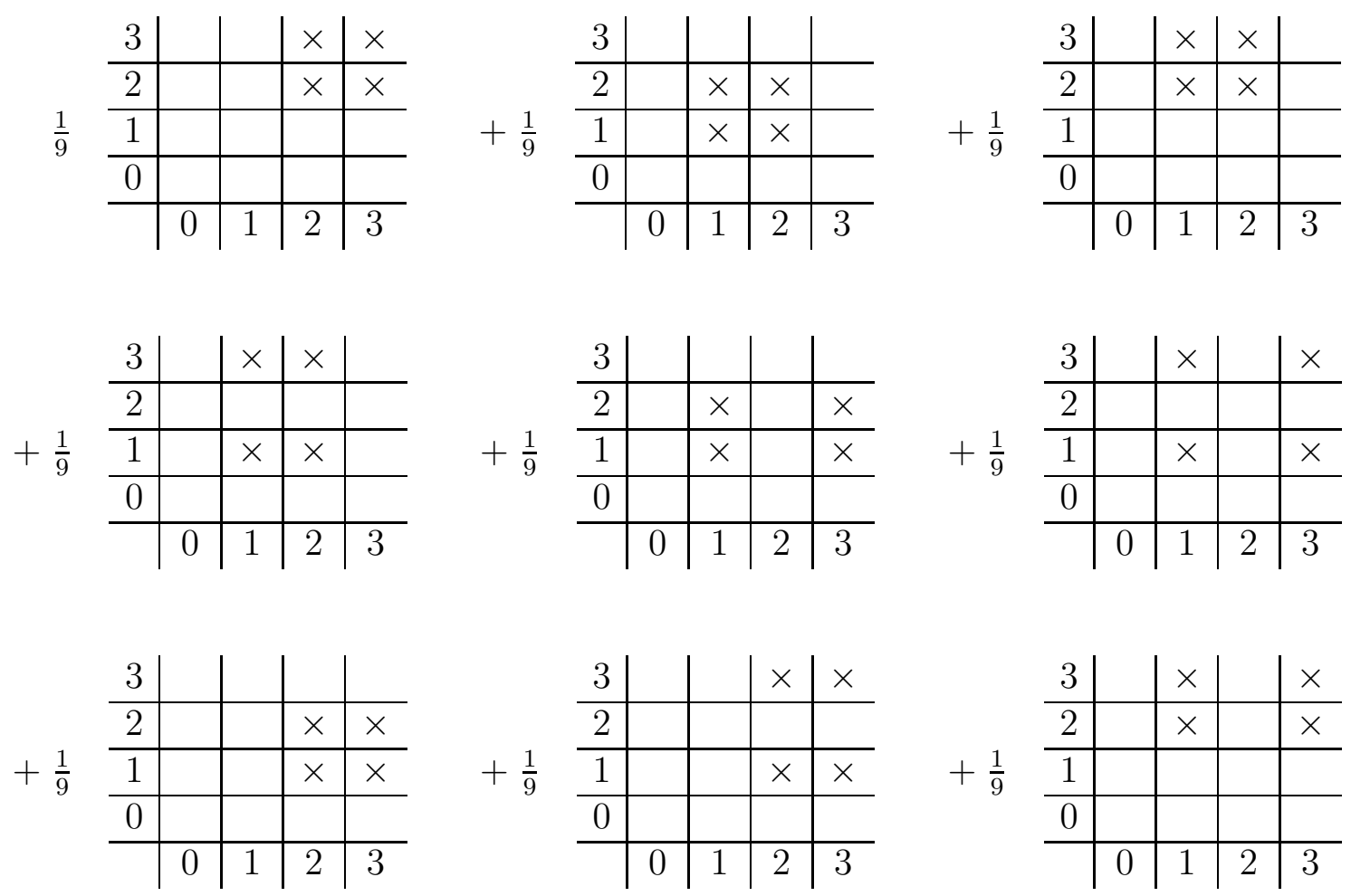

In decomposing $\varrho_{I}^{9}$ as done above, each contributing site from the decomposers appears the same number of times ( 4 in this case) and each decomposer contributes with equal weight (1/9 in this case); then, since the decomposers have $N_{I}=4$, each contributing site appears with weight $4 \times 1 / 36=1 / 9$ in the resulting convex decomposition. From Proposition 1 we know the state $\varrho_{I}^{9}$ is PPT and, as a convex combination of separable states, it is separable.

- $\quad$ Case $N_{I}=8$

With $\mathcal{C}_{0}$ and $\mathcal{R}_{0}$ completely empty there are nine possible states, which can be obtained 
directly from the above state $\varrho_{I}^{9}$ with $N_{I}=9$ by eliminating one element, i.e.

\begin{tabular}{c|c|c|c|c}
3 & & $\times$ & $\times$ & $\times$ \\
\hline 2 & & $\times$ & $\times$ & $\times$ \\
\hline 1 & & & $\times$ & $\times$ \\
\hline 0 & & & & \\
\hline & 0 & 1 & 2 & 3
\end{tabular},

\begin{tabular}{c|c|c|c|c}
3 & & $\times$ & $\times$ & $\times$ \\
\hline 2 & & $\times$ & $\times$ & $\times$ \\
\hline 1 & & $\times$ & & $\times$ \\
\hline 0 & & & & \\
\hline & 0 & 1 & 2 & 3
\end{tabular}

\begin{tabular}{l|l|l|l|l}
3 & & $\times$ & $\times$ & $\times$ \\
\hline 2 & & $\times$ & $\times$ & $\times$ \\
\hline 1 & & $\times$ & $\times$ & \\
\hline 0 & & & & \\
\hline & 0 & 1 & 2 & 3
\end{tabular}

etc.

By exchanging columns and rows as explained in Remark 5, all of these nine possible states are unitarily equivalent to the state

\begin{tabular}{|c|c|c|c|c|c|}
\hline & 3 & & $\times$ & $\times$ & $x$ \\
\hline & 2 & & $\times$ & & $x$ \\
\hline$\varrho_{I}^{8}:$ & 1 & & $\times$ & $x$ & $x$ \\
\hline & 0 & & & & \\
\hline & & 0 & 1 & 2 & 3 \\
\hline
\end{tabular}

Therefore in order to study these lattice states, it is sufficient to see whether $\varrho_{I}^{8}$ is separable or not. From Proposition 1 we know $\varrho_{I}^{8}$ is PPT; moreover, it can be convexly decomposed as follows:

\begin{tabular}{c|c|c|c|c}
3 & & $\times$ & $\times$ & $\times$ \\
\hline 2 & & $\times$ & & $\times$ \\
\hline 1 & & $\times$ & $\times$ & $\times$ \\
\hline 0 & & & & \\
\hline & 0 & 1 & 2 & 3
\end{tabular}
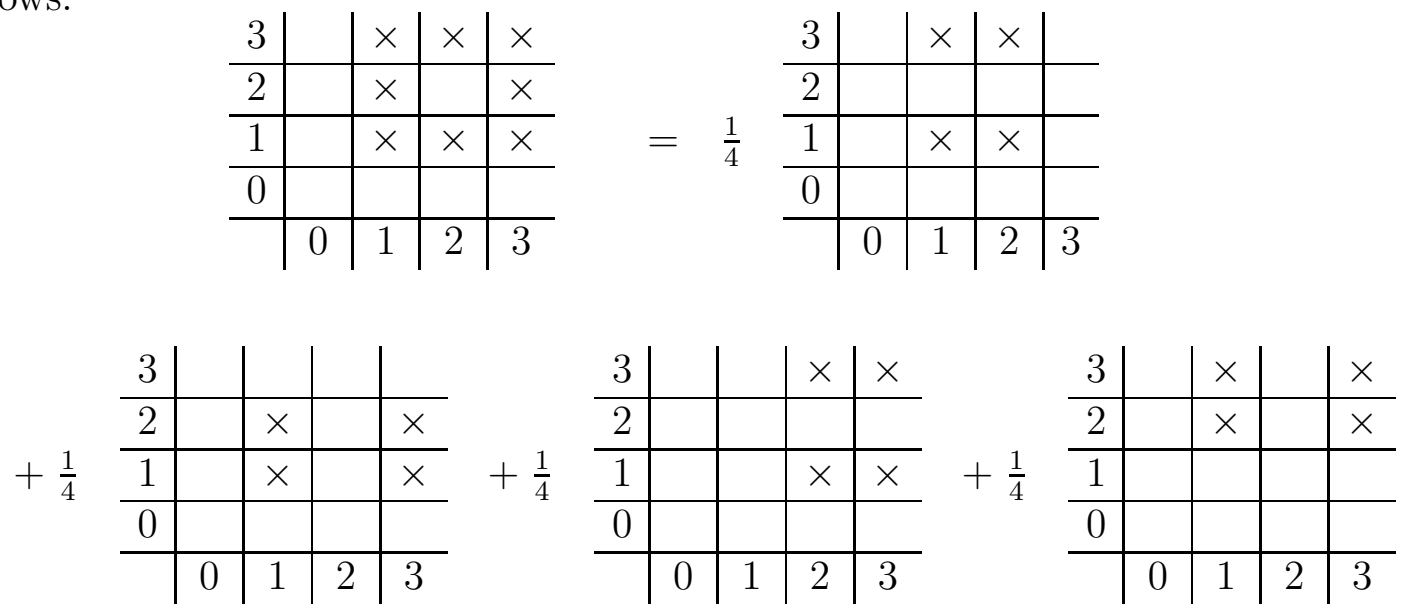

In the above decomposition of $\varrho_{I}^{8}$ each contributing site appears with weight $1 / 4$ in two different PPT decomposers of rank-4; therefore, in the resulting convex combination its weight is $2 \times 1 / 16=1 / 8$. Again, $\varrho_{I}^{8}$ is a mixture of separable states and thus separable itself.

- Case $N_{I}=6$

We have to fit six elements into nine points of the square lattice $L_{16}$ (excluding row $\mathcal{R}_{0}$ and column $\mathcal{C}_{0}$ ). According to Proposition 1, in order for the states to be PPT there cannot be more than two elements in each of the three free columns or rows, i.e. all states such as the 
following are NPT:

\begin{tabular}{l|l|l|l|l}
3 & & $\times$ & $\times$ & \\
\hline 2 & & $\times$ & & \\
\hline 1 & & $\times$ & $\times$ & $\times$ \\
\hline 0 & & & & \\
\hline & 0 & 1 & 2 & 3
\end{tabular}

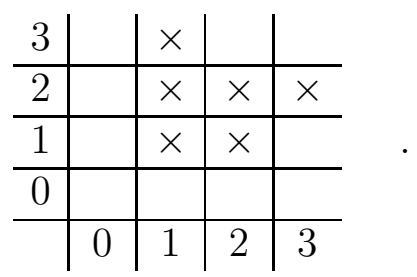

From Proposition 1 it also follows that all states with only two elements in each row (column) but no empty column (row) except for $\mathcal{C}_{0}\left(\mathcal{R}_{0}\right)$ are NPT, as, for instance, the following ones:

\begin{tabular}{l|l|l|l|l}
3 & & $\times$ & $\times$ & \\
\hline 2 & & $\times$ & & $\times$ \\
\hline 1 & & $\times$ & $\times$ & \\
\hline 0 & & & & \\
\hline & 0 & 1 & 2 & 3
\end{tabular}

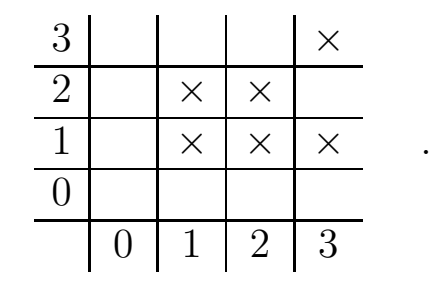

So the only possible way to construct PPT states $\varrho_{I}$ with $N_{I}=6$ and the row $\mathcal{R}_{0}$ and column $\mathcal{C}_{0}$ completely empty, is by putting two elements in each row (column) and leaving another column (row) empty, i.e. lattice states such as $\varrho_{I}^{6}$\begin{tabular}{r|r|r|r|r}
3 & & $\times$ & $\times$ & \\
\hline 2 & & $\times$ & $\times$ & \\
\hline 1 & & $\times$ & $\times$ & \\
\hline 0 & & & & \\
\hline & 0 & 1 & 2 & 3
\end{tabular} and all those in its equivalence class obtained by exchanging columns (rows) among themselves by unitary rotations and row into columns by means of the flip operator as described in Remark 5 .

This state was already showed to be separable in [12, where an explicit decomposition had to be worked out; using the strategy of above, the proof is now much simpler. Indeed, the state $\varrho_{I}^{6}$ can be written as follows:

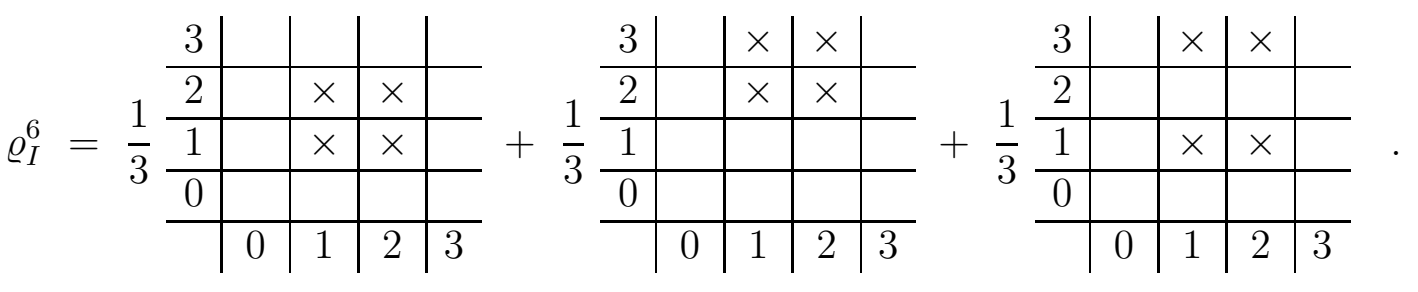

In the above decomposition of $\varrho_{I}^{6}$ each element appears in two different rank-4 lattice states, so that its total weight in the resulting decomposition is $2 \times 1 / 12=1 / 6$. Thus, since $\varrho_{I}^{6}$ is a convex combination of PPT states with $N_{I}=4$, it is separable. Therefore all PPT lattice states with $N_{I}=6$ and a column and row which are completely empty are separable because they are unitarily equivalent to $\varrho_{I}^{6}$. 


\subsection{Lattice States Classification}

From Remarks 1, the only lattice states whose entanglement structure is not under control are those with $N_{I}=6$ and $8 \leq N_{I} \leq 14$. We now show that the case $N_{I}=6$ can also be completely controlled.

As in the proof of Proposition 4, the clue is Remark 4: lattice states can be grouped into equivalence classes, each element in a class being obtainable from any of its partners in that class by the local action of unitaries. Members of a class share the same entanglement properties.

This allows us to consider without restriction those graphs where the largest number of sites, that we shall denote by $n_{0}$, contributing to $I$ by a column are in $\mathcal{C}_{0}$ from bottom to top. We can thus proceed by distinguishing various cases.

- $\quad$ Case $n_{0}=4$

The remaining two contributing sites will necessarily be located on some of the other columns thus violating condition (4): all lattice states with $n_{0}=4$ are thus NPT-entangled.

- $\quad$ Case $n_{0}=3$

1. If any of the sites of $\mathcal{R}_{3}$ contribute to $I$, then the corresponding lattice state violates (4) and is NPT-entangled.

2. If $\mathcal{R}_{3} \bigcap I$ is empty and one of the rows $\mathcal{R}_{0,1,2}$ contributes with more than 1 site to $I$, (41) is again violated and the lattice state is NPT-entangled.

3. If $\mathcal{R}_{3} \bigcap I$ is empty and the 3 rows $\mathcal{R}_{0,1,2}$ contribute with only 1 site to $I$ and there is a column, say $\mathcal{C}_{\alpha}, \alpha \neq 0$, with only 1 site contributing to $I$, then the sufficient condition in Proposition 1 applies to $\mathcal{C}_{\alpha}$ and $\mathcal{R}_{3}$. All these lattice states are thus PPT entangled.

4. If $\mathcal{R}_{3} \bigcap I$ is empty and there is a column (which we can always suppose to be $\mathcal{C}_{1}$ ) with 2 elements in $I$, the remaining contributing site makes these states violate the PPTness condition in Proposition 1. These lattice states are thus NPT-entangled. In fact, the row passing through the last contributing site, let it be $\mathcal{R}_{\beta}$, intersects $\mathcal{C}_{0}$ in one site and $\mathcal{C}_{1}$ in none, whence $\left(\mathcal{C}_{1} \cup \mathcal{R}_{\beta}\right) \bigcap I$ contains 4 sites.

5. If $\mathcal{R}_{3} \bigcap I$ is empty and the 3 sites of $I$ from the rows $\mathcal{R}_{0,1,2}$ are on a same column $\mathcal{C}_{\alpha}$, $\alpha \neq 0$, then we are in the situation of Proposition 4 . These lattice states are thus separable.

- $\quad$ Case $n_{0}=2$

1. If one of the rows $\mathcal{R}_{2,3}$ contributes with more than 1 site to $I$, condition (4) is violated and the state $\varrho_{I}$ is NPT-entangled. 
2. If the rows $\mathcal{R}_{2,3}$ altogether contribute to $I$ with 1 site at the most, then there will be at least one of the rows $\mathcal{R}_{0,1}$ intercepting $I$ in not less than three sites. In this case, using the flip operator as in Remark 4.3, rows can be turned into columns and we are back with either $n_{0}=3$ or $n_{0}=4$.

3. If each of the rows $\mathcal{R}_{2,3}$ intersects $I$ in only one point and these two elements lie on a same column, again (4) is violated and $\varrho_{I}$ is NPT-entangled. Indeed, the two elements can always be thought to be along the column $\mathcal{C}_{1}$ which cannot then contain other sites from $I$. Therefore, (44) is violated by any remaining contributing site exactly as in point 4 when $n_{0}=3$. The following is a representative of such a possibility:

\begin{tabular}{l|l|l|l|l}
3 & & $\times$ & & \\
\hline 2 & & $\times$ & & \\
\hline 1 & $\times$ & & & $\times$ \\
\hline 0 & $\times$ & & $\times$ & \\
\hline & 0 & 1 & 2 & 3
\end{tabular}.

4. The only remaining case is thus represented by

\begin{tabular}{c|c|c|c|c}
3 & & & & $\times$ \\
\hline 2 & & & $\times$ & \\
\hline 1 & $\times$ & $\times$ & & \\
\hline 0 & $\times$ & $\times$ & & \\
\hline & 0 & 1 & 2 & 3
\end{tabular}. This state is
separable as it can be decomposed into $3 \mathrm{PPT}$ lattice states $\rho_{I}$ with $N_{I}=4$. As in Proposition 4, we indicate the weights with which the various graphs contribute to the decomposition:

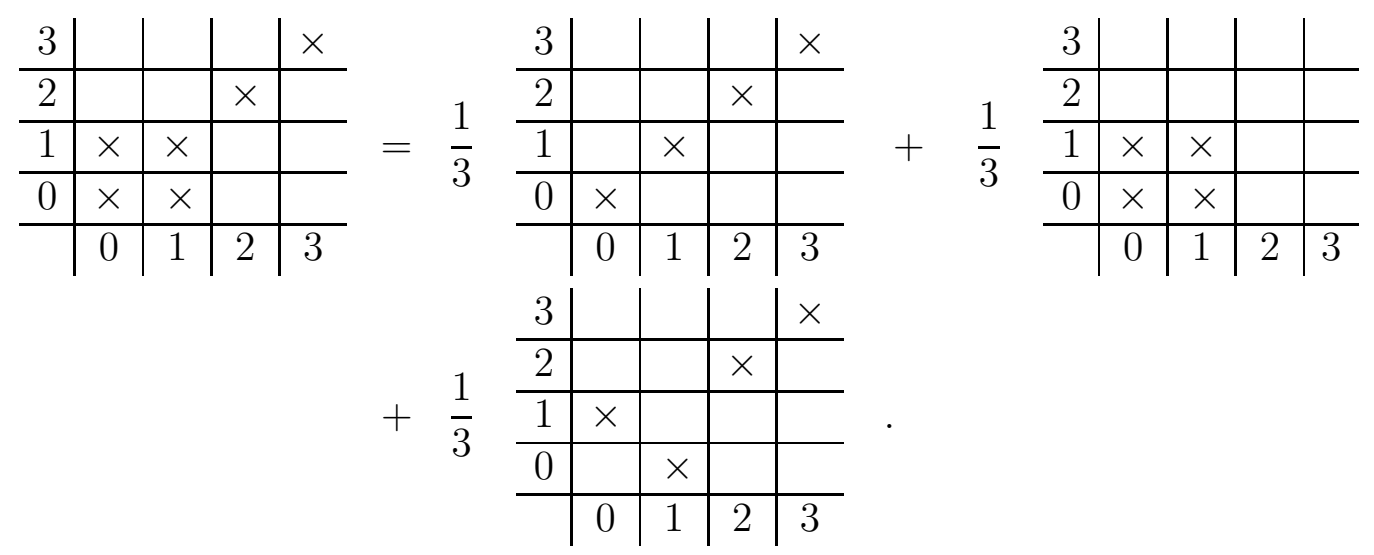

\section{Conclusions}

Because of the difficulty in finding appropriate entanglement witnesses, the detection of bipartite entanglement and its qualification as bound or free still represents a challenge from 
the point of view of mathematical physics. Of course, the issue at stake is the lack of a structural characterization of positive, but not completely positive maps.

In order to deepen our actual understanding of positivity of linear maps, in particular with respect to their indecomposability and the corresponding phenomenon of bound entanglement, it is still important to provide classes of states together with maps that are able to detect the different aspects of their entanglement.

To this end, in this paper we studied a class of bipartite states, called lattice states, each party consisting of 2-qubit systems, which are nicely associated with subsets $I$ of points on a square lattice with 16 elements. By using a suitably adapted extended reduction criterion, we exposed certain relations between bound entanglement and the geometric pattern of the subsets $I$ labeling the lattice states. More precisely, based on an existing complete characterization of which of the lattice states are PPT, we showed that

1. all PPT lattice states labeled by a subset $I$ such that there exist a column $\mathcal{C}_{\alpha}$ and a row $\mathcal{R}_{\beta}$ of the lattice contributing to $I$ with only one point different from $(\alpha, \beta)$ are entangled.

2. If $(\alpha, \beta) \notin I$, the extended reduction criterion reproduces a result already obtained with a different indecomposable positive map. If $(\alpha, \beta) \in I$, new bound entangled lattice states are witnessed.

3. All PPT lattice states labeled by a subset $I$ that entirely excludes a column and a row are separable.

However, a thorough classification of the class of lattice states still requires stronger criteria than the ones presented in this paper. Indeed, the positive map $\Phi_{V}$ (7) is unfortunately not an exhaustive entanglement witness; for instance, it is indecisive in the following cases of PPT lattice states. The first three examples are with $\kappa=0$ for some $(\alpha, \beta) \in I$, while the last one, with $N_{I}=11$, is a geometric pattern which cannot be controlled by any of the methods employed in this paper:

$$
\begin{aligned}
& N_{I}=8: \begin{array}{c|c|c|c|c}
3 & & \times & \times & \times \\
\hline 2 & & \times & & \times \\
\hline 1 & & \times & & \times \\
\hline 0 & \times & & &
\end{array}, \quad N_{I}=9: \quad \begin{array}{l|l|l|l|l}
3 & & \times & \times & \times \\
\hline 2 & & \times & & \times \\
\hline 1 & & \times & \times & \times \\
\hline 0 & 0 & 1 & 2 & 3
\end{array},
\end{aligned}
$$

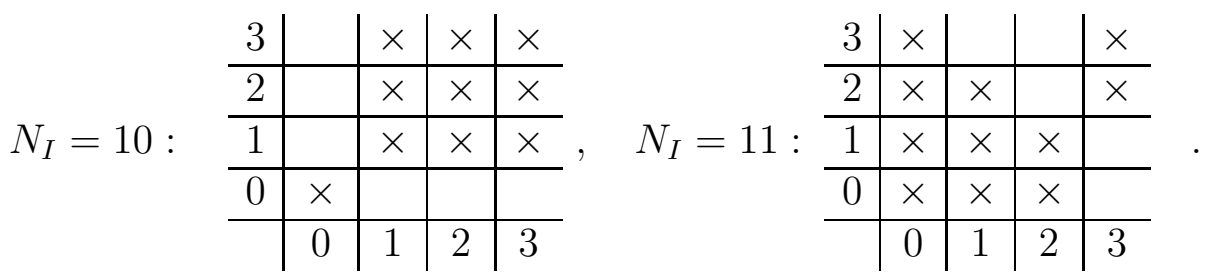




\section{References}

[1] M. Horodecki, P. Horodecki, R. Horodecki, in: Quantum Information: An Introduction to Basic Theoretical Concepts and Experiments, Springer Tracts in Modern Physics (July 2001)

[2] M.D. Choi Linear Algebra Appl. 10, 285 (1975)

[3] S.L. Woronowicz Rep. Math. Phys. 10, 165 (1976)

[4] E. Störmer Acta Math. 110, 233 (1963)

[5] D. Chruscinski, A. Kossakowski, Phys. Rev. A 74, 022308 (2006)

[6] D. Chruscinski, A. Kossakowski, On the structure of entanglement witnesses and new class of positive indecomposable maps, quant-ph/0606211

[7] S. Albeverio, K. Chen, S.M. Fei, Phys. Rev. A 68, 062313 (2003)

[8] M. Horodecki, P. Horodecki, Phys. Rev. A 59, 4206 (1999)

[9] H.-P. Breuer, Phys. Rev. Lett. 97, 080501 (2006)

[10] W. Hall Construction of indecomposable positive maps based on a new criterion for indecomposability, quant-ph/0607035

[11] F. Benatti, R. Floreanini, M. Piani Phys. Lett. A 326, 187 (2004)

[12] F. Benatti, R. Floreanini, M. Piani Open Sys. and Information Dyn. 11, 325 (2004)

[13] K. S. Gibbons, M. J. Hoffmann, W. K. Wootters, Phys. Rev. A 70, 062101 (2004)

[14] A. O. Pittenger, M. H. Rubin Mutually unbiased bases, generalized spin matrices and separability, quant-phys/0308142

[15] A. O. Pittenger, M. H. Rubin, Journal of Physics A 38, 6005 (2005)

[16] E.F. Galvao, Phys. Rev. A 71, 042302 (2005)

[17] P. Horodecki, M. Lewenstein, G. Vidal, I. Cirac, Phys. Rev. A 62, 032310 (2000) 